Check for updates

Cite this: Mater. Chem. Front., 2020, 4, 715

Received 14th December 2019, Accepted 15th January 2020

DOI: $10.1039 / c 9 q m 00760 a$

rsc.li/frontiers-materials

\section{Stimuli-responsive behaviors of organic charge transfer cocrystals: recent advances and perspectives}

\author{
Lingjie Sun,$\dagger$ Fangxu Yang, $\dagger$ Xiaotao Zhang (D) * and Wenping Hu (D) *
}

Organic cocrystals, as an intelligent design strategy of functional materials, have received extensive attention in the scientific community over recent years. The presence of diverse molecular arrangements and rich intermolecular interactions, in particular intrinsic charge transfer in organic cocrystals, depicts such materials as ideal systems to control and tailor their physical properties for stimuli-responsive applications. However, as a continuous emerging research field, a systematic review to summarize this promising application area remains unexplored. Herein, we focus on recent research progress and potential advantages of organic cocrystals in the field of stimuli response. First, an overview of the basic characteristics of organic charge transfer cocrystals is discussed. Subsequently, the stimuli-responsive behaviors of organic cocrystals under mechanical force, solvent, acid-base, light and heat stimuli are highlighted. Lastly, the future developments and prospects of these materials in a stimuli-responsive field are outlined.

\section{Introduction}

Stimuli-responsive materials alter their physicochemical properties such as shape, color, and luminescence, under external stimuli, which would be desirable candidates for applications

Tianjin Key Laboratory of Molecular Optoelectronic Science, Department of Chemistry, School of Science, Tianjin University \& Collaborative Innovation Center of Chemical Science and Engineering (Tianjin), Tianjin 300072, China.

E-mail: zhangxt@tju.edu.cn,huwp@tju.edu.cn

$\dagger$ These authors contributed equally to this work. in memories, sensors, informational displays and encryption. ${ }^{1-5}$ Stimulation styles are usually diverse, which can be force, ${ }^{6}$ light, ${ }^{7,8}$ heat, ${ }^{9}$ solvent, ${ }^{10}$ acid, ${ }^{11,12}$ and so on. Stimuli-responsive characteristics can be achieved when the material undergoes an internal structural change under external stimuli, thereby creating new physical properties. ${ }^{13-16}$ Molecular materials connected by noncovalent bonds, where this interaction is relatively weak and is sensitive to external changes, are promising for designing stimuliresponsive functions. ${ }^{17,18}$ However, till date, extensive investigations have been performed on the stimuli-responsive behaviors of

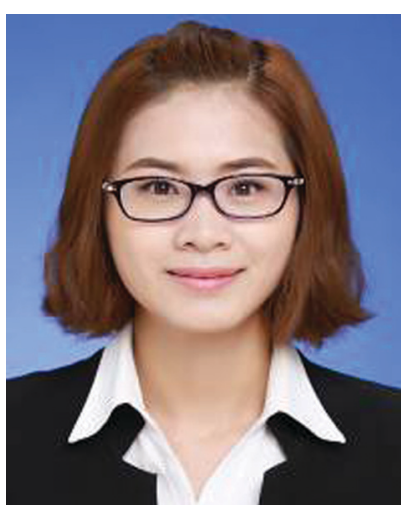

Lingjie Sun
Lingjie Sun grew up in Tangshan, Hebei Province, P. R. China. In 2017, she received her MSc degree in the Department of Chemistry, School of Science, Tianjin University and in the Collaborative Innovation Center of Chemical Science and Engineering (Tianjin). Since September 2017, she has been a PhD student in the same department (Supervisor: Prof. Wenping $\mathrm{Hu}$ ). Her interests are in design, characterization and optoelectronic

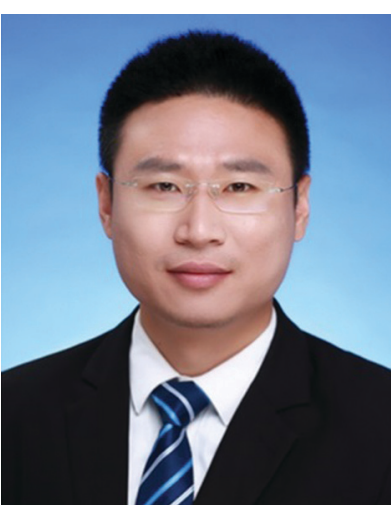

Fangxu Yang
Fangxu Yang is an associate professor in the Department of Chemistry, School of Science, Tianjin University. He received his $P h D$ degree from the Institute of Chemistry, Chinese Academy of Sciences (ICCAS) in 2016, and then joined Tianjin University as a postdoctoral student of Collaborative Innovation Center of Chemical Science and Engineering (Tianjin) until 2019. His research interests focus on molecular materials, crystals and optoelectronic devices. 
single-component molecules, while multiple component molecules have been studied to a lesser extent, ${ }^{19-22}$ which could be attributed to the uncontrolled proportion and inhomogeneous distribution of amorphous multi-component solids, and their resulting unpredictable and non-replicable performances. ${ }^{23}$

Ordered assembled molecular solids composed of multiple components, also known as organic cocrystals, are assembled by noncovalent bonds, such as halogen bonds, hydrogen bonds, $\pi-\pi$, and charge transfer interactions, ${ }^{24,25}$ which exhibit a clear stacking structure, providing a platform to unveil the structure-property relationship at the molecular level. ${ }^{26,27}$ Among all, charge transfer cocrystals assembled via donor species with strong electron donating capacity and acceptor species possessing strong electron-withdrawing ability ${ }^{28}$ have proven beneficial in order to achieve high-quality cocrystals with regular shapes. ${ }^{29}$ In addition, the charge transfer effects of organic cocrystals indicate that freely moving electrons are transferred from the donor to the acceptor material, which can induce electron delocalization over the entire supramolecular structure, thus exhibiting different photoelectric properties from its constituent materials and generating new properties, which provide vast opportunity to explore novel types of appealing applications. ${ }^{30,31}$ For example, metallic conductivity, ${ }^{32}$ ferroelectricity, ${ }^{33,34}$ nonlinear optics, ${ }^{35,36}$ ambipolar charge transport, ${ }^{37,38}$ and many other fascinating phenomena ${ }^{39,40}$ have been created by engineering charge transfer effect in organic cocrystals. More importantly, the degree of charge transfer could be detected by spectral characterization, and undergoes changes in response to external stimuli, which, in turn, helps to design and understand the target functionality. In this regard, we systematically summarize and introduce the stimuli-responsive behavior of organic charge transfer cocrystals in this review (Fig. 1). First, the molecular arrangements of organic cocrystals, preparation methods, and common donor as well as acceptor molecules are discussed. Second, the stimuli-responsive behavior of organic cocrystals under mechanical force, solvent, acid-base, light and heat stimuli are highlighted. Finally, future outlooks are provided. We hope this review adds to already existing knowledge in this field as well as open new research pathways toward the development of novel material systems for stimuli-responsive applications.

\section{Basic properties of organic charge transfer cocrystals}

\subsection{Common packing modes}

As known, the packing patterns in organic cocrystals play an important role in the control of their bulk properties. ${ }^{31}$ In the crystal structure of the cocrystal, there is a clear stoichiometric distribution ratio between donor and acceptor molecules, where the value may be of equal (i.e. $1: 1$ ) or non-equal (i.e. $2: 1,3: 1,3: 2$ and $4: 3$ ) ratios. ${ }^{41,42}$ Among all, the pattern ratio of $1: 1$ stacked cocrystals, which includes segregated and mixed stacking, presents the most common structures. For segregated stacking

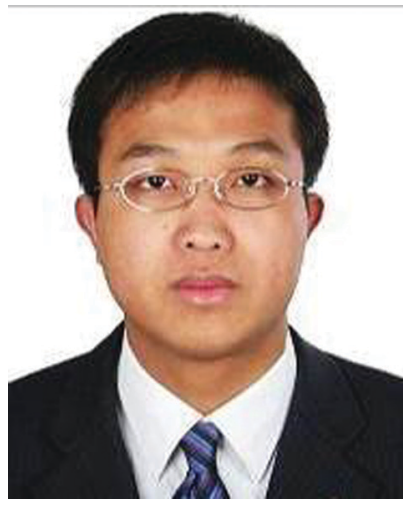

Xiaotao Zhang
Xiaotao Zhang is an associate Professor at Tianjin University. He was born in 1982 in Henan province, China. He received his $\mathrm{PhD}$ degree from the Institute of Chemistry, Chinese Academy of Sciences in 2012 after he got his MSc degree (2007) at Zhejiang University. His research work includes the design and synthesis of novel organic semiconductors, the fabrication and characterization of organic optoelectronic devices.

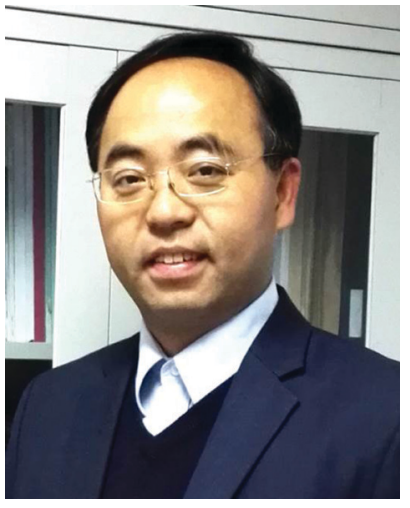

Wenping $\mathrm{Hu}$ is a Professor of School of Science, Tianjin University, and a Cheung Kong Professor of the Ministry of Education, China. He received his PhD from the Institute of Chemistry, Chinese Academy of Sciences (ICCAS) in 1999. Then he joined Osaka University and Stuttgart University as a research fellow of the Japan Society for the Promotion of Sciences and Alexander von Humboldt, respectively. In 2003 he worked in Nippon Telephone and Telegraph (NTT), and then joined in ICCAS again as a full professor. He worked for Tianjin University in 2013 and was promoted to vice president of the university in 2016. He served as a Visiting Scholar at the Department of Chemistry, Stanford University in 2007, and a Visiting Professor at the Department of Chemistry, National University of Singapore in 2013. He focuses on organic optoelectronics, and has published 4 books (Organic Optoelectronics for Wiley etc.) and more than 500 peer-reviewed papers with citations $\sim 18000$ times ( $H$ index $=68$ ). He worked as an Associate Editor of Polymer Chemistry (RSC, 2013-2016), and is a member of the editorial boards for several journals (e.g., Adv. Energy Mater., Adv. Electron. Mater., Nano Res., Sci. Bull., Sci. China Mater.). 


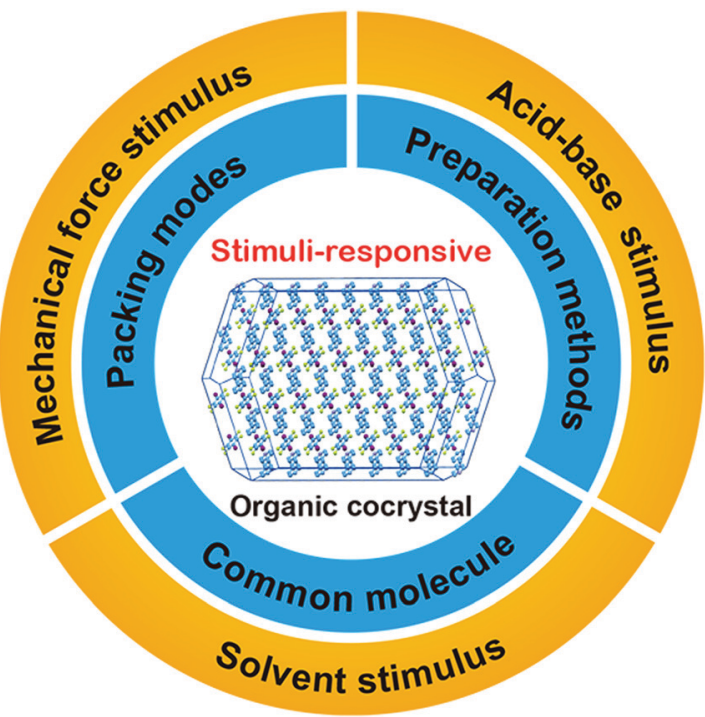

Fig. 1 An overview of this review addressing the key points of organic cocrystals towards stimuli-responsive applications.

cocrystals, the donor and acceptor molecules are arranged in adjacent molecular columns (-DDDD- and -AAAA-). ${ }^{43}$ These adjacent molecular columns connect with intermolecular forces to form a three-dimensional network structure. However, the donor and acceptor molecules of the mixed stacking cocrystals are alternately arranged in a similar molecular column (-DADA-), while the adjacent molecular columns are connected to each other forming a tightly packed stack (Fig. 2). ${ }^{35,44}$ It is worth noting that the donors and acceptors are stacked along their respective columns in segregated stacking cocrystals, allowing most of these systems to display preference for a large degree of charge transfer and thus exhibit metallic properties as expressed following the example of TTF-TCNQ co-crystals. ${ }^{45}$ By contrast, the donor molecules are inserted by acceptors into the mixed stacking cocrystals, and most of those systems are prone to a small degree of charge transfer, and thus, they have been regarded as insulators/semiconductors. ${ }^{46}$

\subsection{Preparation methods}

Similar to the habits of monomolecular crystals, the morphologies of cocrystals can be divided into micro/nano and bulk

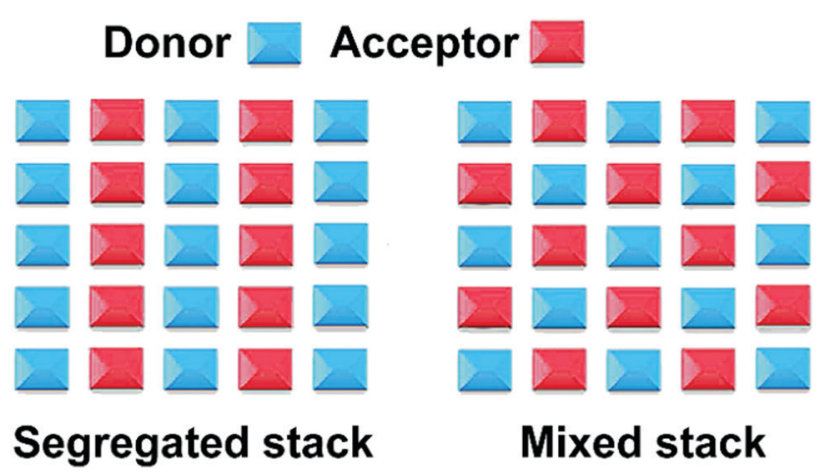

Fig. 2 Packing modes of cocrystals in a 1:1 stoichiometric ratio. ${ }^{30}$ (Reprinted with permission from the Royal Society of Chemistry. Copyright 2018.) crystals based on their sizes, which are affected by the methods of preparation. ${ }^{47-52}$ Till date, the liquid-phase and vapor methods are being commonly employed to prepare cocrystals. ${ }^{47,53}$ In general, some liquid-phase methods, such as drop-casting and liquid-liquid interfacial precipitation methods, can facilitate the rapid identification of donors and acceptors, and their assembly and nucleation to form micro/nano-sized cocrystals (Fig. 3a). ${ }^{54-56}$ The advantages of these types of methods include simplicity, rapidity and cost-effectiveness, and these micro/nano-sized cocrystals not only can be used to analyze the internal driving force and growth orientation, ${ }^{57}$ but are also suitable for the construction of high-performance devices such as OFET and OLET ${ }^{58}$ Notwithstanding, some liquid-phase methods such as solvent evaporation and diffusion method offer the opportunity to design and develop large-sized cocrystals following a slow solvent evaporation process. ${ }^{59}$ These methods have proven to be time-consuming, but the prepared cocrystals are able to grow into well-ordered structures with high quality, which can be used for the X-ray single-crystal structure characterization and anisotropic studies of physical properties. With respect to the vapor methods, which mainly refer to the physical vapor transport (PVT), these methods are useful to grow cocrystals for insoluble compounds (Fig. 3b) ${ }^{60-62}$ Additionally, when the intermolecular force of the cocrystal is relatively strong, the resulting products can be prepared via either the vapor or liquid-phase methods. For instance, the perylene-TCNQ cocrystals are grown along the [001] direction with strong charge transfer interactions, which are prepared via dropcasting ${ }^{63}$ or PVT $^{41}$ producing nanowires (Fig. 3c-e). Moreover, the solid-state grinding approach is also an important method to prepare organic cocrystals. ${ }^{64,65}$ This preparation method is

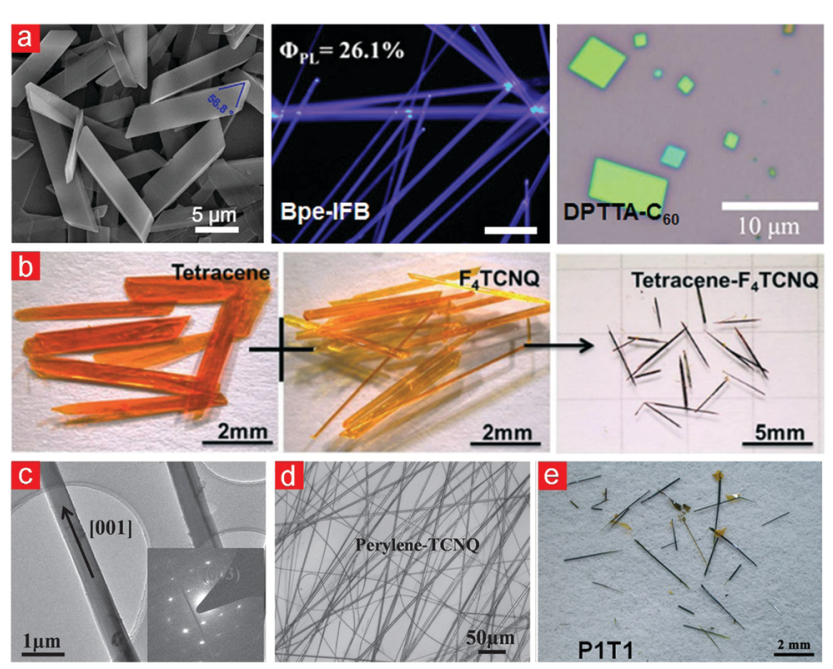

Fig. 3 (a) Cocrystals grown by liquid-phase methods. ${ }^{54-56}$ (Reprinted with permission from American Chemical Society. Copyright 2017, 2015, 2013.) (b) Cocrystals grown by vapor methods. ${ }^{61}$ (Reprinted with permission from Wiley-VCH. Copyright 2017.) (c) TEM and SAED results of perylene-TCNQ cocrystals, and (d) perylene-TCNQ cocrystals grown by drop-casting. ${ }^{63}$ (Reprinted with permission from Wiley-VCH. Copyright 2015.) (e) Perylene-TCNQ cocrystals grown by PVT. ${ }^{41}$ (Reprinted with permission from American Chemical Society. Copyright 2014.) 
not only simple and effective, but can also effectively expand the molecular system for synthesizing cocrystals, especially for those molecules with poor solubility.

\subsection{Common donors and acceptors of charge transfer cocrystals}

Typically, donor and acceptor molecules are constituent units of cocrystals that play important roles in determining the physicochemical properties of cocrystals. Compared to donors, the types of acceptor molecules used for cocrystal preparation are relatively limited as shown in Fig. 4. They are mainly concentrated in tetracyanoquinodimethane (TCNQ) and its derivatives, naphthalenediimide (NDI) and perylenediimide (PDI), and their derivatives, fullerene $\left(\mathrm{C}_{60}, \mathrm{C}_{70}\right), 1,2,4,5$-tetracyanobenzene (TCNB) and tetrafluoroterephthalonitrile (TFP). ${ }^{53}$ Moreover, the chemical structures of the acceptor molecules are relatively simple. For example, TCNQ and its derivatives as well as fullerene can assemble with appropriate donors to form charge transfer cocrystals with good electrical properties, while TCNBbased charge transfer cocrystals usually display luminescent properties. ${ }^{15}$ In order to rationally predict the functions of cocrystals based on these acceptors, it is crucial to choose a suitable donor material. This is because the choice of donor not only affects the selected preparation method, but also affects the stacking structure of cocrystals, which ultimately determines the bulk properties of the cocrystals. Therefore, some representative donor materials (tetrathiafulvalene and their derivatives, acene and their derivatives as well as stilbene and their derivatives) that provide guidance in the rational design of functional cocrystals are discussed in the following section.

Various studies have shown that tetrathiafulvalene (TTF)-like molecules have good planarity, which interact with TCNQ molecules to form cocrystals. Most of the prepared cocrystals exhibit segregated stacking structures and a relatively strong charge transfer effect that occurs between the donors and acceptors,

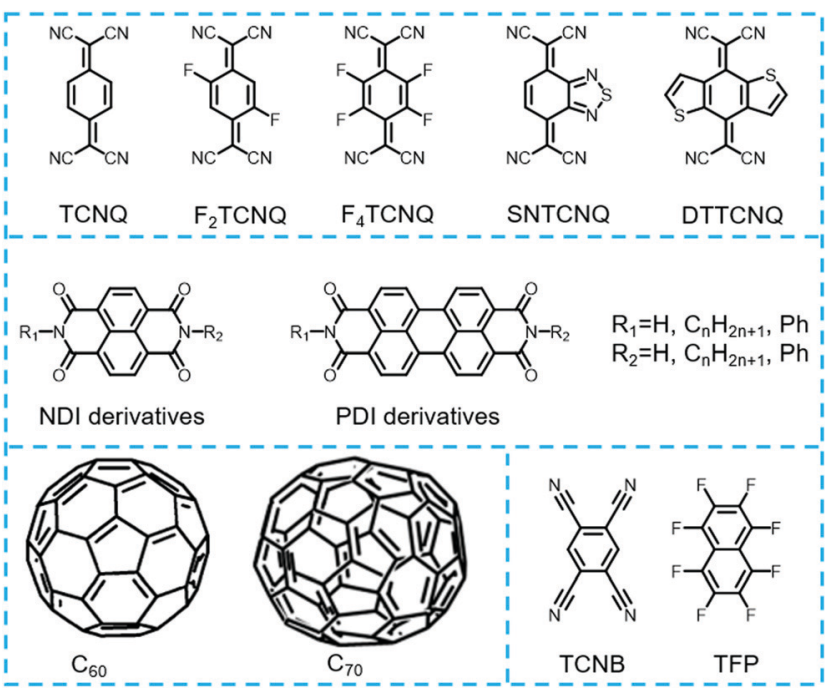

Fig. 4 Molecular structures of typical acceptors of cocrystals. leading to cocrystals exhibiting good electrical conductivity. Typically, the investigation of TTF-like charge transfer cocrystal has been kicked off since A. J. Heeger et al. discovered TFF-TCNQ cocrystals exhibiting extraordinary conductivity, ${ }^{45}$ which is absent in their monomers and can be used as an organic metal electrodes for high-performance devices based on all-organic materials. Subsequently, it is unexpectedly found that (BEDT-TTF) ( $\mathrm{F}_{2}$ TCNQ) (bis(ethylenedithio)-tetrathiafulvalene, BEDT-TTF; 2,5-difluoro-7,7,8,8-tetracyanoquinodimethane, $\mathrm{F}_{2} \mathrm{TCNQ}$ ) cocrystals possess ambipolar charge transport behaviors under lowtemperature conditions, ${ }^{66}$ and the research boom of TTFbased donor-based ambipolar charge transport materials has been developed. Among them, the charge-carrier mobilities of the (BEDT-TTF)-TCNQ cocrystal at room temperature were up to $(0.6-3) \times 10^{-2} \mathrm{~cm}^{2} \mathrm{~V}^{-1} \mathrm{~s}^{-1}$ for electrons and $(0.4-2) \times$ $10^{-2} \mathrm{~cm}^{2} \mathrm{~V}^{-1} \mathrm{~s}^{-1}$ for holes. ${ }^{67}$ Goetz et al. showed that the $\beta$-polymorph of DBTTF-TCNQ (DBTTF: dibenzothiatetrathiafulvalene) cocrystal displayed ambipolar transport behaviors with mobilities of $\mu_{\text {hole }}=0.1 \pm 0.07 \mathrm{~cm}^{2} \mathrm{~V}^{-1} \mathrm{~s}^{-1}$ and $\mu_{\text {electron }}=$ $0.03 \pm 0.02 \mathrm{~cm}^{2} \mathrm{~V}^{-1} \mathrm{~s}^{-1} .^{68}$ In addition, the charge transfer cocrystals formed by TTF-like molecules not only impart good electrical properties, but also help to reduce the band gap of the cocrystal, even develop it into the near-infrared absorption region, obtaining a high-performance near-infrared photothermal conversion material. More recently, Wang et al. obtained a DBTTF-TCNB cocrystal following a simple evaporation method with strong charge transfer interactions between DBTTF and TCNB molecules. ${ }^{69}$ Theoretical and experimental studies fully demonstrated the charge transfer nature of the ground and excited states, which results in the generation of cocrystals with a narrow band gap of $1.3 \mathrm{eV}$. This corresponded to a significant red-shifted near-infrared (NIR) absorption $(904 \mathrm{~nm})$. At the same time, the radiation transition process of the excited state of DBTTF-TCNB cocrystal was suppressed by solid-state fluorescence quenching and underwent rapid non-radiative decay. Therefore, the DBTTF-TCNB cocrystal exhibited high-performance photothermal conversion characteristics with high cyclic stability. Moreover, the prepared cocrystal materials also show a great potential for photothermal imaging in real-time and time-resolved response (Fig. 5).

The acenes and their derivative molecules can form stable charge transfer complexes with classical acceptors, such as TCNB, TCNQ and fullerene. As shown in Fig. 6, small-molecularweight acenes such as naphthalene, anthracene and pyrene can assemble with TCNB to form charge transfer cocrystals, and those cocrystals usually display tunable emission properties. For instance, the naphthalene-TCNB cocrystals demonstrate blue emission; ${ }^{70}$ the phenanthrene-TCNB cocrystals demonstrate green emission; ${ }^{71}$ the pyrene-TCNB cocrystals demonstrate orange emission, ${ }^{44}$ and so on. By increasing the molecular weight or the $\pi$-conjugation of acenes, the charge transport in the acenes can be improved. ${ }^{72}$ It was found that perylene or corannulene molecules can act as donors and interact with TCNQ to form cocrystals with good electrical properties, showing excellent application prospect in the fields of photodetector and 


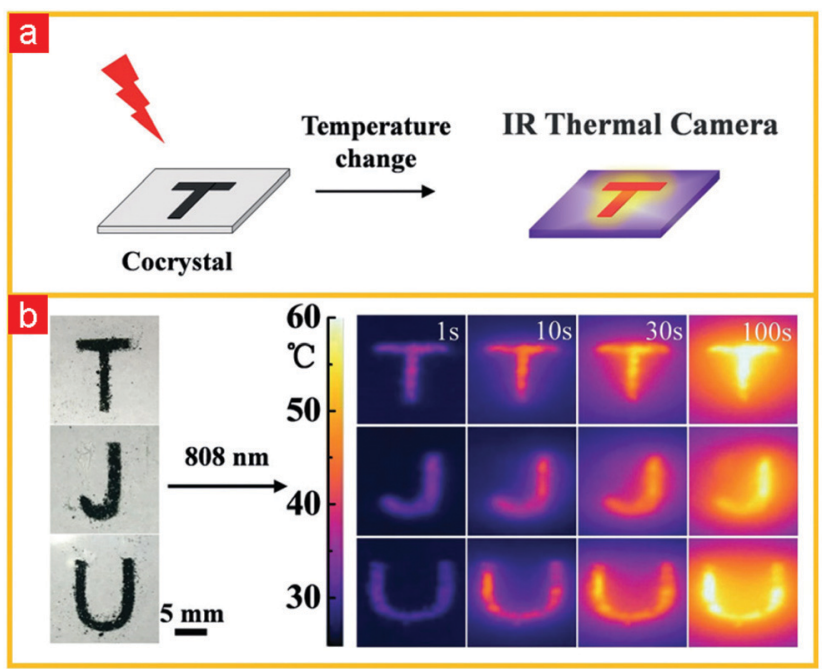

Fig. 5 (a) Diagram of photothermal imaging process. (b) IR camera images of cocrystal-patterned letters TJU. ${ }^{69}$ (Reprinted with permission from Wiley-VCH. Copyright 2018.)

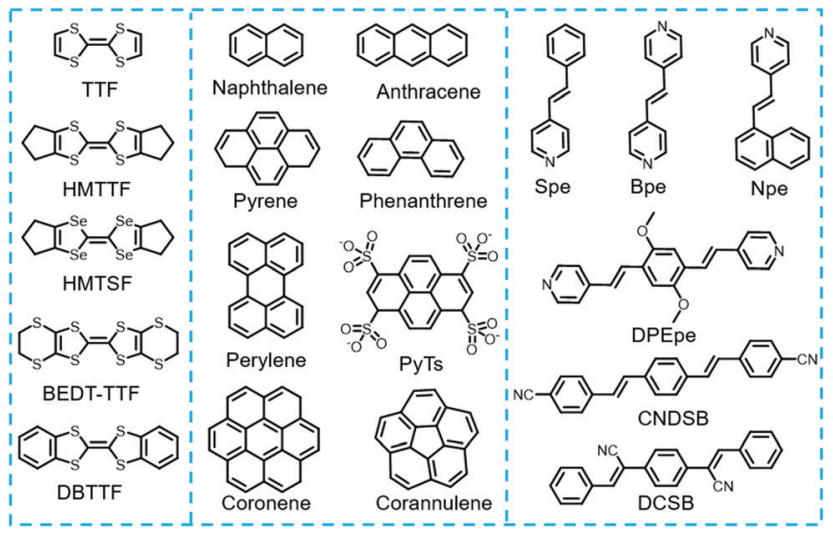

Fig. 6 Molecular structures of typical donors of cocrystals.

OFET. In 2015, Wu et al. demonstrated that the coronene-TCNQ cocrystal exhibited an n-type transistor behavior with a mobility of $0.02 \mathrm{~cm}^{2} \mathrm{~V}^{-1} \mathrm{~s}^{-1}$, an $I_{\text {on }} / I_{\text {off }}$ ratio of $1 \times 10^{5}$ and a very small threshold voltage of $0.7 \mathrm{~V}$, which are much better than those of its acceptor. ${ }^{73}$

The stilbene-like molecules are one well-documented class of organic donor materials, which can be combined with small molecular acceptors to obtain organic cocrystals with multiple functions, showing promising optoelectronic applications in the fields of optical waveguide properties, ${ }^{74,75}$ nonlinear optics $^{35}$ pure organic room-temperature phosphorescent properties, ${ }^{76}$ organic light-emitting transistors (OLET) ${ }^{77}$ and stimuli-responsive properties, ${ }^{78}$ etc. More recently, Li et al. have reported that the cocrystals of naphthylvinylpyridine (Npe) and tetrafluoroterephthalic acid (TFA) exhibited photoactuating behaviors due to the occurrence of photoinduced [2+2] cycloaddition reactions, providing a detailed understanding of the relationship between their self-assembled structures and photoactuating performances. ${ }^{79}$

\section{Stimuli-responsive behaviors of organic charge transfer cocrystals}

\subsection{Mechanical stimuli}

The pressure-sensitive materials refer to those with the changes in some physicochemical properties under the stimulation of external mechanical forces such as grinding force, shearing force, tensile force and static pressure, which have been extensively researched in recent years. ${ }^{80-87}$ At present, organic substances, metal complexes and polymer materials have been developed to exhibit controlled fluorescence characteristics under the stimulation of these forces. ${ }^{88,89}$ So far, related investigation of organic substances has become more popular due to their advantages including low cost, flexibility and largescale production. ${ }^{90}$ Organic cocrystals are composed of different molecules with non-covalent bonds, structures or intermolecular forces that are modifiable under mechanical stimuli, which ultimately affects the functioning of the materials. ${ }^{91-95}$ Herein, the research on external force stimulation based on charge transfer cocrystals mainly focuses on isotropic static pressure and anisotropic grinding forces, and is discussed in this section. With respect to the isotropic static pressure, which is usually conducted on a diamond anvil cell device, silicone oil or the mixed organic solution (such as the mixture of methanol and ethanol) is widely employed as the pressure-transmitting medium. Note that the molecular orbitals of charge transfer cocrystals are related to the highest occupied molecular orbitals (HOMO) of the donor, and the lowest unoccupied molecular orbitals (LUMO) of the acceptor, thus a narrowed band gap will be obtained, which gives rise to the red-shifts of the ultravioletvisible (UV-vis) adsorption and emission spectra compared to their single components. ${ }^{28}$ Generally, when applying the static pressure on the organic charge transfer cocrystals, the unidirectional pressure can lead to enhanced intermolecular interactions and the degree of the charge transfer, causing the rearrangement of the HOMO of donor and the LUMO of acceptor, which will affect the spectral properties of the cocrystals. ${ }^{96}$ Specifically, the UV-vis adsorption and emission spectra are gradually red-shifted to longer wavelength regions than those of pristine cocrystals with the increase in pressure, while the bulk properties of the cocrystals will be restored to the initial state when completely removing the external pressure. Therefore, the pressure-induced charge transfer cocrystals offer the opportunity to adjust the photoemission continuously and reversibly, providing a simple and effective method to control the cocrystal functions in situ. For example, Xu et al. prepared a mixed stacking charge transfer cocrystal composed of 9-acetylanthracene (9ACA) and tetrafluoroterephthalonitrile (TFP) in a 2:1 ratio, which exhibited continuous redshifts in its emission spectra with a large range (from 490 to $620 \mathrm{~nm}$ ) with the pressure increasing. ${ }^{97}$ The well-grown 9ACA-TFP cocrystals were placed on the diamond anvil device to study the in situ tunable properties of the fluorescence emission under ambient pressure. In the cocrystal compression process, as shown in Fig. 7a, the fluorescence colors of the 9ACA-TFP cocrystal changed from aquamarine blue to green, orange and 


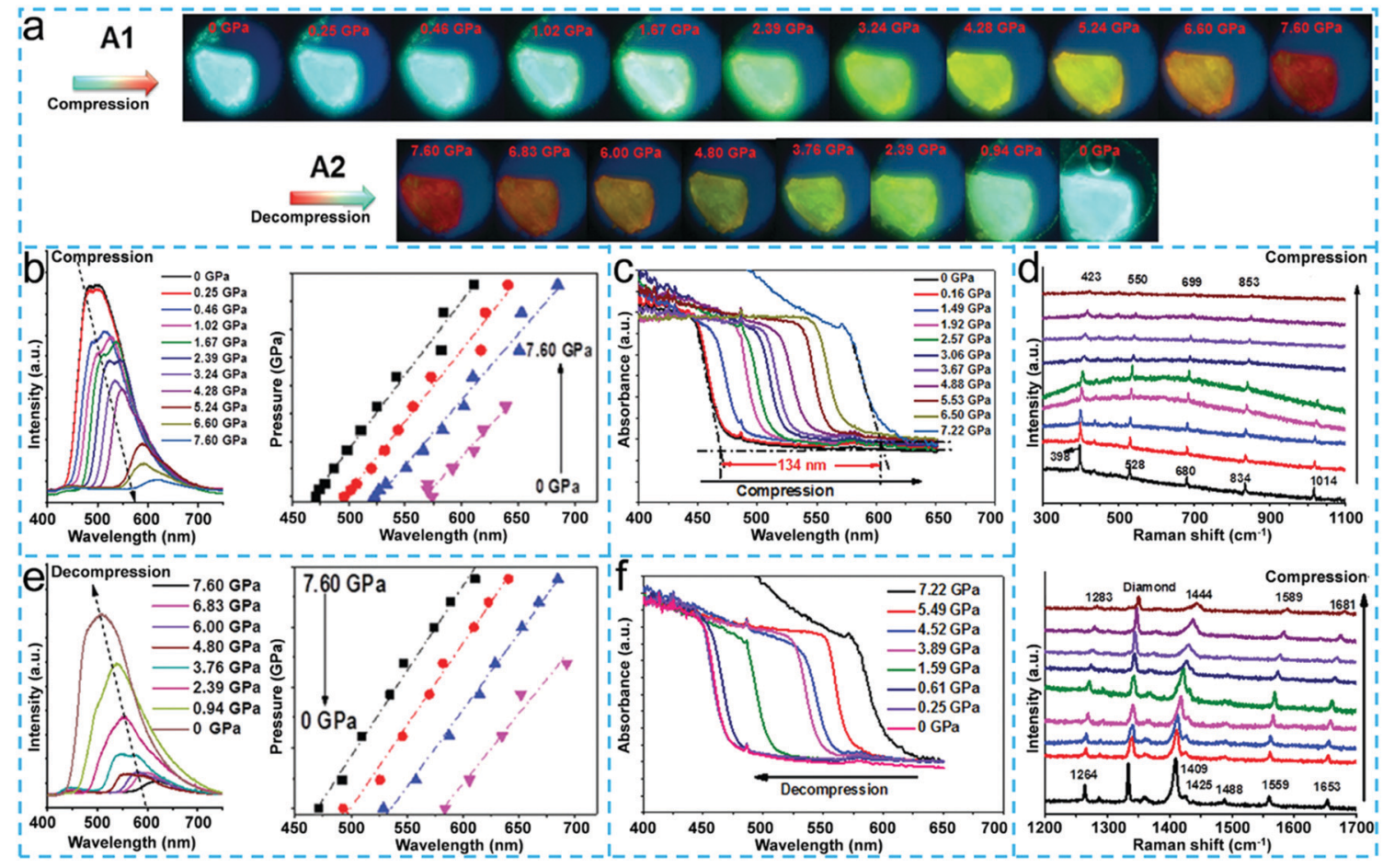

Fig. 7 (a) Fluorescence images (A1 and A2) of the 9ACA-TFP co-crystal during compression and decompression. Fluorescence spectra and the plots of the emission wavelength with pressure (b). The UV-visible absorption spectra (c). Raman spectra (d) of the 9ACA-TFP co-crystal during compression. Fluorescence spectra and the plots of the emission wavelength with pressure (e). The UV-visible absorption spectra (f) of the 9ACA-TFP co-crystal during decompression. ${ }^{97}$ (Reprinted with permission from the Royal Society of Chemistry. Copyright 2018.)

finally to red during the gradual increase in pressure. From the fluorescence spectra collected under high pressure (Fig. 7b), the intensity of the emission peak gradually weakens and moves toward the long wavelength with the increase in pressure. In addition, the emission wavelength and the pressure increase linearly. The authors speculated that these results may be due to the enhanced degree of intermolecular charge transfer under high pressure. To further demonstrate this opinion, the authors impressively performed in situ UV-vis absorption spectroscopy and in situ Raman spectroscopy to analyze the intermolecular forces of the cocrystal under external pressure. In situ UV-vis absorption spectroscopy showed that the absorption spectrum was red-shifted $(134 \mathrm{~nm})$ as a whole and the cocrystal band gap was narrowed (from $2.63 \mathrm{eV}$ to $0.59 \mathrm{e} \mathrm{V}$ ) during the cocrystal compression process (Fig. 7c), indicating that the interplanar distance was increased and the intermolecular interactions were increased under high pressure. The HOMO of the donor molecule and the LUMO of the acceptor molecule were rearranged, and the cocrystal band gap was thus narrowed. Meanwhile, the frequency regions of the Raman vibration mode of the 9ACA-TFP cocrystal were shifted to the high wavenumber during the compression process (Fig. 7d), which further indicated that the bond lengths of the cocrystal molecule became shorter and the strength of the intermolecular interactions were enhanced with the increase in pressure, consistent with the results of in situ UV-vis absorption spectroscopy. However, the results of the cocrystal fluorescence colors, the fluorescence spectra and the UV-vis absorption spectra are opposite to those of the compression process releasing the pressure (Fig. 7a, e and f), indicating that photoemission properties of the cocrystal material can achieve in situ reversible regulation under the external pressure stimulation.

On the other side, the exertion of grinding forces on organic materials is also mostly investigated due to its easy operation, which does not need some special devices, and the mortar in the laboratory can meet the requirements. Unlike static pressure, the grinding force is an anisotropic force. Generally, the crystal state of the organics can be deformed into amorphous solids, and the molecular arrangements are destructed when they are operated by mechanical grinding and hence leading to the change in bulk properties. For instance, Yan et al. demonstrated that the photoemission band of (2,5-diphenyloxazole) (1,4-diiodotetrafluorobenzene) cocrystals moved from $393 \mathrm{~nm}$ to $384 \mathrm{~nm}$ accompanied by the appearance of a shoulder peak at $374 \mathrm{~nm}$ and a slight increase in the luminescence intensity, displaying a change in fluorescence properties upon grinding. ${ }^{83}$ They performed the powder X-ray diffraction (XRD) measurement to explain this phenomenon and found that several diffraction peaks of original cocrystals disappeared after grinding, suggesting the formation of amorphous phase, which accounted for the change in fluorescence. Undoubtedly, the external mechanical forces can perturb the molecular arrangement of cocrystal materials, so research on materials that respond to multiple mechanical stimuli will be more attractive. Impressively, Tian et al. found that isotropic static pressure and anisotropic grinding forces have different effects 


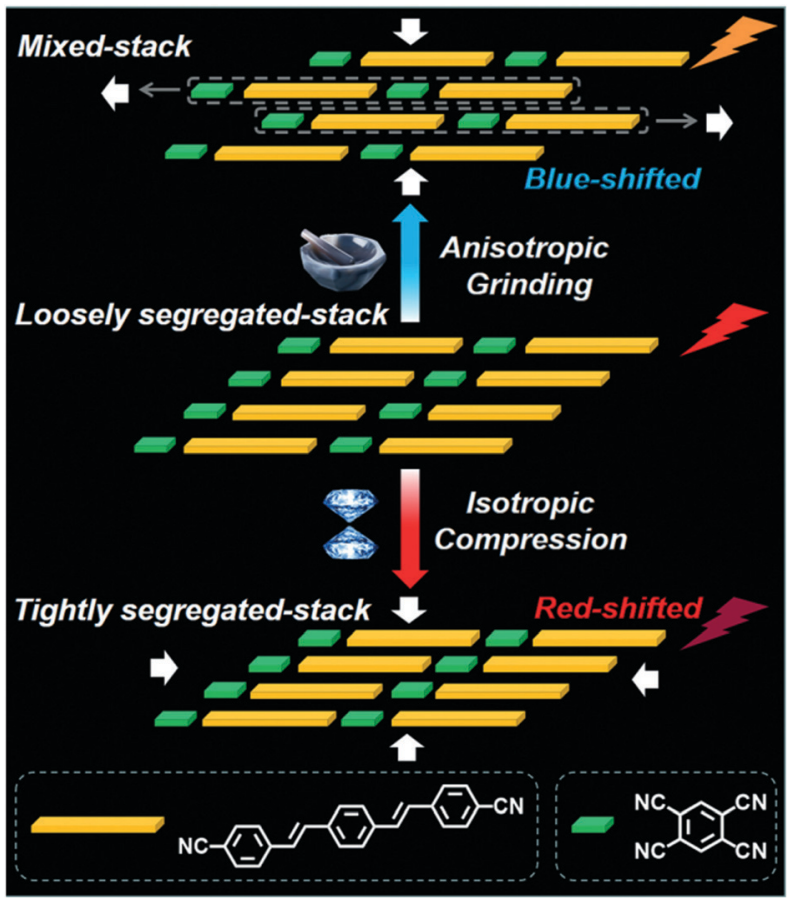

Fig. 8 Transition modeling schematic of the molecular stacking mode in CT-R cocrystals under different mechanical force stimuli. ${ }^{22}$ (Reprinted with permission from Wiley-VCH. Copyright 2018.)

on fluorescence properties of the charge transfer cocrystal (Fig. 8). ${ }^{22}$ In detail, they prepared a block-shaped red chargetransfer cocrystal by a solvent evaporation process, also called CT-R, in which 1,4-bis- $p$-cyanostyrylbenzene (CNDSB) severed as a donor and TCNB as an acceptor. When the anisotropic grinding force was applied, the emission spectrum of CNDSBTCNB cocrystals was blue-shifted, and the photoluminescence quantum yield (PLQY) was increased from $13.0 \%$ to $22 \%$. The diffraction peak of powder XRD after grinding is completely different from that of the pristine cocrystal, indicating that the CT-R cocrystal may undergo a structural reorganization during the grinding process. However, the CT-R cocrystal red-shifted in both the absorption spectrum and the fluorescence spectrum under the isotropic static pressure. Besides, the intensity of the fluorescence emission decreased with the increase in pressure, different from those of the grinding process. Moreover, when the external pressure is removed, the fluorescence behavior of the cocrystal material could be restored to the initial state. Therefore, we know that the CT-R cocrystal exhibits significant differences in fluorescence behavior under different external force stimuli. According to the analysis of the single-crystal structure, the CNDSB-TCNB cocrystals exhibited two polymorphs, that is, one was a 1:1 mixed stacking crystal phase called CT-O, and the other was the previously mentioned $4: 3$ segregated stacking for CT-R. The PLQY value of the CT-O crystal phase was $26.2 \%$, and its fluorescence spectrum was very close to that of the ground CNDSB-TCNB cocrystal, and the powder XRD pattern of the CNDSB-TCNB cocrystal could correspond well to those of CT-O. Thus, these data indicated that a structural reorganization occurred from the segregatedstacked to the mixed-stacked under the exertion of an anisotropic grinding force, resulting in the weakening of $\pi-\pi$ and charge transfer interactions among CNDSB and TCNB and, thus, weak exciton coupling between the pairs of neighboring CNDSB and CT transition strength, which contributed to the blue-shift of fluorescence spectrum and the improved PLQY. However, the hydrostatic pressure induced the molecules to form a tighter packing structure and enhanced $\pi-\pi$ and charge transfer interactions, eventually leading to red-shift emission.

\subsection{Solvent stimuli}

Solvents are of paramount importance in crystal growth by liquid phase methods, which are typically related to morphology, size and polymorph of the crystals. ${ }^{26}$ Moreover, in the process of multiple-component cocrystallization, a rational choice of solvent can also effectively adjust the molar ratio of donors to acceptors. ${ }^{63}$ Even when the intermolecular forces between the donor and acceptor are insufficient to form a well-ordered structure, solvent molecules can enter the frameworks to promote efficient crystallization. ${ }^{99}$ Therefore, the bulk phase properties of the cocrystal can be regulated by selecting different solvents during the crystal growth process. ${ }^{100}$ For instance, Zhang et al. demonstrated that the solvent accommodation not only tuned the structure-stacking modes and, hence, affected the degree of charge transfer of the cocrystals, but also switched off electron-transport behaviors of coroneneF $_{4}$ TCNQ cocrystals $(1: 1)$ in an ambient atmosphere, which implied that organic charge transfer cocrystals might provide an effective way to control the functions on demand by a solvent-mediated method. ${ }^{101}$ However, the solvent-stimulated cocrystal materials herein are well-grown cocrystal molecules that can be invaded by an external solvent molecule or an internal solvent molecule and can be removed from the cocrystal, which have potential applications in the areas of multi-color photoluminescence switching, memory and smart materials. For example, Park et al. prepared a weakly chargetransfer organic cocrystal composed of a distyrylbenzene-based donor (4M-DSB) and a dicyanodistyrylthiophene-based acceptor (Thio-Y), where a loosely packed molecular arrangement was designedly obtained as a result of the noncentrosymmetric chemical structure of molecule A1 (Fig. 9a). ${ }^{98}$ The intended loosely packed molecular organization of the cocrystal could be characterized by distinct free volume and relatively weak intermolecular interactions (D-A interaction: $3.5 \AA$, $-\mathrm{CN} \cdots \mathrm{HC}-$ interaction: $2.6 \AA$, Fig. 9b), allowing for a reversible-phase alteration in response to external stimuli. The pristine D1-A1 spin-coated cocrystal films emitted red fluorescence in the case of nonpolar solvent and polar protic solvent vapor annealing (SVA), while it exhibited blue light emission once stimulated by polar aprotic solvents (Fig. 9c). They explained that the cocrystal exhibited mixed packing in the former case, but when a polar aprotic solvent was used, the packing mode was closer to the structure of the donor material in the demixed phase, which indicated that the crystal packing structure could undergo a controlled transformation under the condition of adopting 


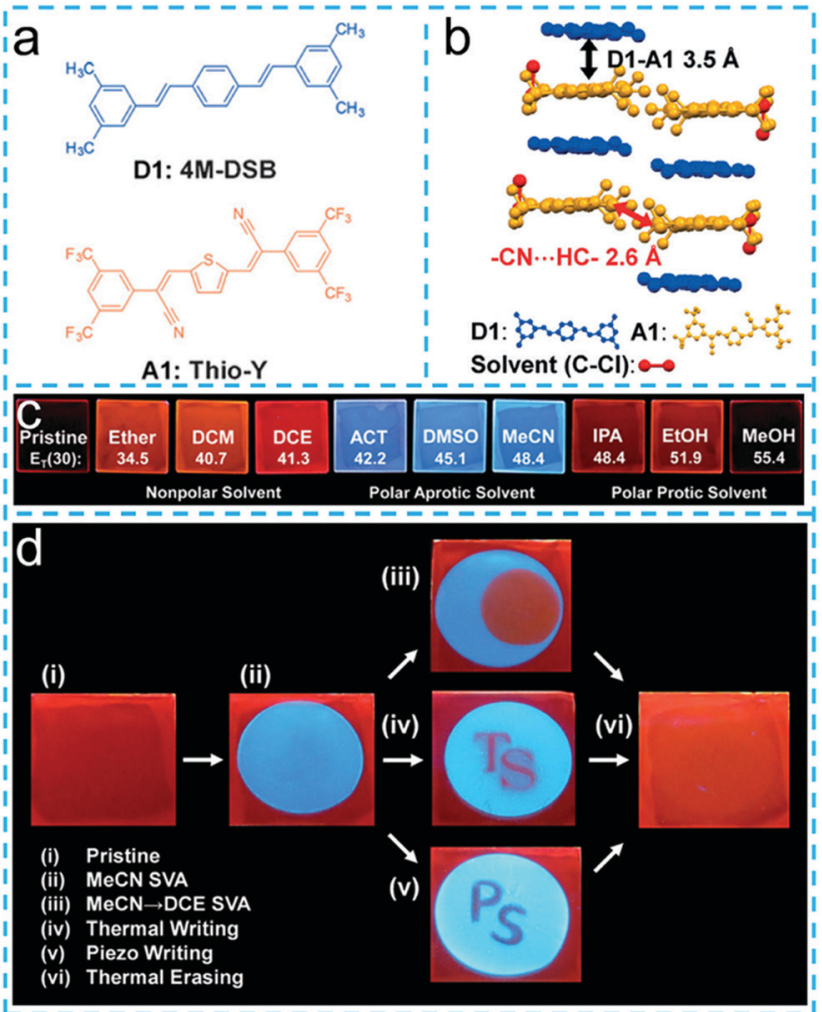

Fig. 9 (a) Chemical structures of the donor (D1: 4M-DSB) and acceptor (A1: Thio-Y). (b) Roll-angle view with information of the D1-A1 $\pi-\pi$ distance (black arrow) and the A1-A1 $-\mathrm{CN}$...HC- distance (red arrow) (c) Digital images of CT spin-coated films taken under $365 \mathrm{~nm}$ UV irradiation. (d) Optical memory demonstration of D1-A1 CT spin-coated film consisting of three components (D1, A1, and PMMA), utilizing various external stimuli. The thermal erasing process that took place after piezo writing exhibited an inevitable stain. ${ }^{98}$ (Reprinted with permission from Wiley-VCH. Copyright 2016.)

suitable solvent, thus affecting fluorescence properties. Based on this feature, they further designed a multistimuli-responsive programmable fluorescence memory system (Fig. 9d), providing important insights for the supramolecular control of intermolecular interactions by the strategic selection of external triggers.

In addition, Hu group also found that the charge transfer cocrystal could undergo macroscopic mechanical bending behaviors under the stimulation of external solvents and clearly demonstrated the crystal bending mechanism at the molecular scale. ${ }^{102}$ They reported a red-emitting solvent-included charge transfer cocrystal (perylene-TCNB)-2THF, also called PT2TC. When the ternary solvated PT2TC cocrystal was placed in the air condition, the THF solvent molecules escaped from the cocrystal, and the fluorescent color changed from red to green. However, when it was again placed in the THF solvent atmosphere, it formed the original three-component cocrystal coupled with mechanical bending behaviors (Fig. 10a). In order to reveal the mechanism for this phenomenon, they performed time-resolved FTIR, absorption and photoluminescence (PL) spectra (Fig. 10b). Studies had shown that after the threecomponent PT2TC cocrystal was placed in air for 10 minutes,

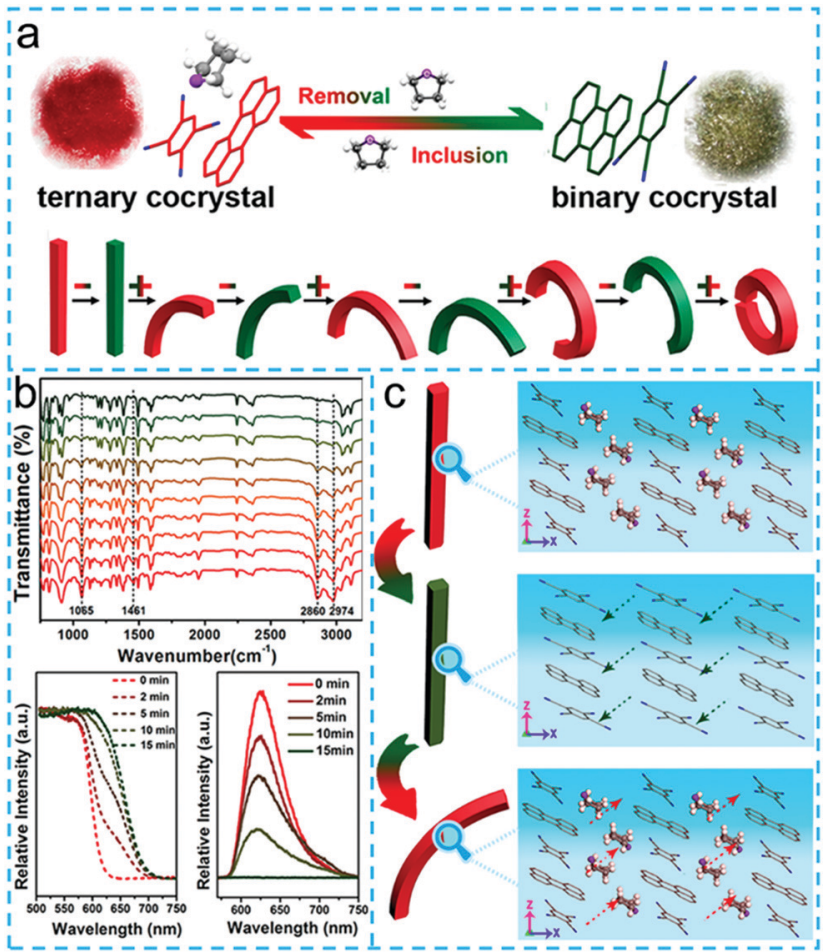

Fig. 10 (a) Reversible structural and color changes between ternary PT2TC and binary PTC (upper). Schematic of mechanical bending of a cocrystal rod ("+" represents the inclusion of THF, " -" displays the removal of THF, below). (b) Time-resolved FTIR spectra, absorption and PL spectra of PT2TC when exposed to air. (c) Schematic of mechanical bending mechanism of PT2TC at the molecular scale. ${ }^{102}$ (Reprinted with permission from American Chemical Society. Copyright 2018.)

the IR bands originated from the constitute unit kept unchanged, but the characteristic IR peaks of the three components (1065, 1461, 2860, and $2974 \mathrm{~cm}^{-1}$ ) disappeared, and the PXRD pattern of the resultant crystals displayed obvious shifts when compared to the initial ternary PT2TC, indicating the change in material composition from the PT2TC into binary PTC when exposed to air. At the same time, its absorption spectrum was red-shifted accompanied by a decrease in emission intensity upon exposure to air, demonstrating that the degree of charge transfer of the THF-excluded PTC was higher than that of PT2TC. As for the changes in the mechanical behavior mentioned, the authors explained that after removal of the THF molecule, a straight rod-like appearance could be maintained due to the rigid crystal structure of the perylene-TCNB cocrystal. However, there are some voids in the perylene-TCNB cocrystal structure, and the strong charge transfer interaction would cause the TCNB to move slightly toward the center of the perylene plane, which was responsible for the fluorescent color change from red to grayish-green. When exposed to THF solvents, the voids in the loose crystals would again be occupied by the new THF molecules. The invasion of the THF molecule would force the TCNB to return to its original position, thereby driving the TCNB to slide between the layers toward the [010] direction (Fig. 10c). Subsequently, the rigidity of PT2TC on the molecular scale was reduced, further favoring the bending. 


\subsection{Acid-base vapor stimuli}

Generally, external acid/base compounds interact with certain components of organic cocrystals, giving rise to the change in the stacking structure or intermolecular forces, which ultimately affects the cocrystals' luminescence behaviour. ${ }^{103}$ For example, Yan et al. used 4-(1-naphthylvinyl)pyridine (Npe) as the donor molecule and 4-benzoylbenzoic acid (BBA) as the acceptor molecule, ${ }^{104}$ in which the donor molecule with the pyridine unit provides the nitrogen atom and hydrogen, and the acceptor molecule offers the oxygen atom in the carboxylic acid and the hydrogen atom on the hydroxide, which are interconnected by intermolecular hydrogen bonds $(\mathrm{OH} \cdots \mathrm{N}$, $1.63 \AA$; $\mathrm{CH} \cdots \mathrm{O}, 2.58 \AA$ A) to form a three-dimensional network in Npe-BBA cocrystal structures. The Npe-BBA cocrystals emitted green fluorescence $(516 \mathrm{~nm})$ under UV light. When it is placed in $\mathrm{a} \mathrm{NH}_{3}$ atmosphere for 30 seconds, the luminescent color changes to blue $(435 \mathrm{~nm})$. The authors speculate that the mechanism may be the deprotonation of pyridine nitrogen atoms of Npe under alkaline conditions. Interestingly, by placing it in an acidic atmosphere, the fluorescent color can be reversible back to the initial state (Fig. 11). Moreover, they also prepared a cocrystal with adjustable fluorescence emission properties under acid-base stimulation. The donor used Npe derivative 4-[2-(4-quinolinyl)vinyl]phenol (qv), and the acceptor adopted tetrafluoroterephthalic acid (a). In an acidic atmosphere of $\mathrm{HCl}$, the qv-a cocrystal has a slight red-shift to the red light region of $597 \mathrm{~nm}$ due to the proton transfer process between the $\mathrm{HCl}$ and the cocrystal, and the fluorescence intensity is greatly enhanced; however, when it is placed in an alkaline atmosphere of $\mathrm{NH}_{3}$, the spectrum blue-shifts back to $517 \mathrm{~nm}$ and the fluorescence intensity is weakened. Notably, reversible transformation of the fluorescent color by acid-base stimulation can be repeated multiple times. This indicates that acid-base stimulation could be an effective tool for regulating the fluorescence emission behavior of organic cocrystals, which provides a new idea and basis for the development of new stimuli-responsive materials and photo sensors.

\subsection{Other stimuli}

Other important stimuli forms such as light and heat are also common means to explore the response characteristics of

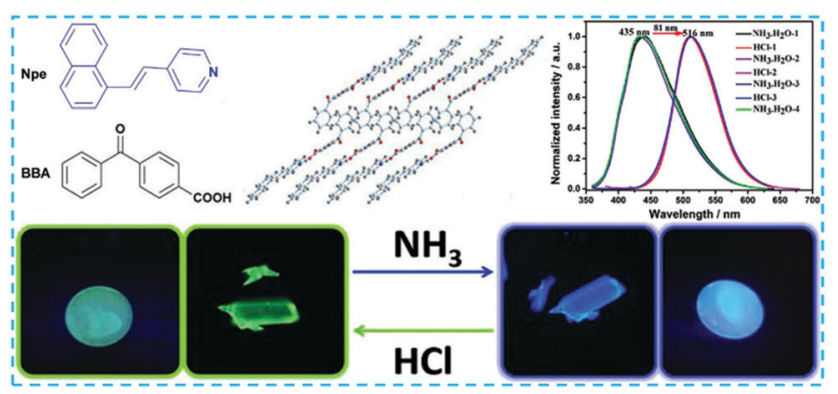

Fig. 11 Changes in luminescence behavior of charge transfer cocrystal under external acid-base vapor stimulation. ${ }^{104}$ (Reprinted with permission from Wiley-VCH. Copyright 2018.)
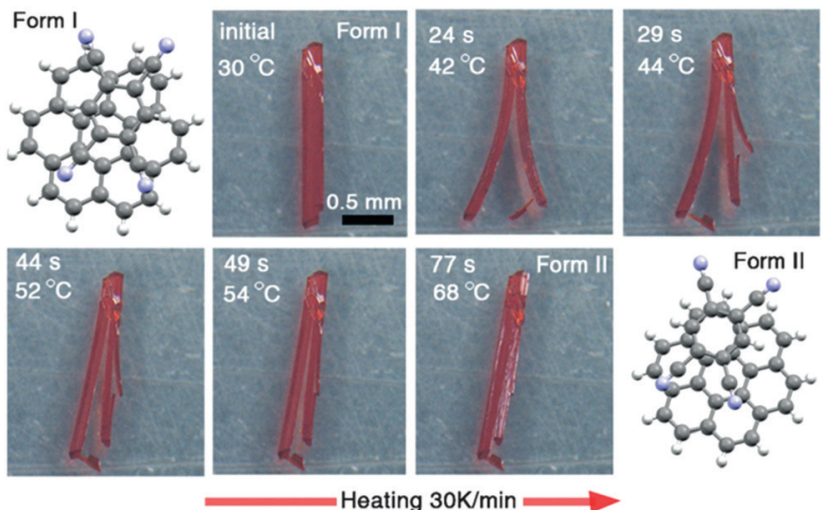

Fig. 12 Remarkable self-healing behavior in coronene-TCNB cocrystals. Images recorded using an optical microscope equipped with a variabletemperature hot stage. ${ }^{13}$ (Reprinted with permission from Wiley-VCH. Copyright 2015.)

organic charge transfer cocrystals. For example, under light stimulation, the resulting photogenerated carriers usually change the conductivity of semiconductor materials, which is the basic principle of photodetectors. ${ }^{105-107}$ At present, this photoelectric conversion phenomenon has been widely reported in organic charge transfer cocrystals. $^{63,72}$ Besides, photomechanical response phenomenon has been discovered in azobenzene cocrystals, which remarkably undergo a crystal-to-crystal cis-trans isomerisation accompanied by large-scale bending. ${ }^{108}$ Moreover, recently, Wang et al. have explored an effective photothermal conversion property in charge transfer cocrystals. ${ }^{69}$ The strong D-A interaction leads to a narrow bandgap with NIR absorption. It was remarkably found that the temperature of cocrystals is sharply increased in a short time using the NIR laser illumination with high photothermal conversion efficiency.

Molecular crystals can have different crystal polymorphs in the molecular packing mode. Heat stimulation is the most important method for tuning the mutual transformation of crystal polymorphs. In general, stable thermodynamic products tend to form at high temperatures, and kinetic products tend to form at low temperatures. This thermally stimulating crystal polymorph transition has been found in organic charge transfer cocrystals. For instance, Liu et al. reported a remarkable self-healing behavior in coronene-TCNB cocrystals (Fig. 12). ${ }^{13}$ The as-prepared cocrystals show an interesting thermo-mechanical response during thermally induced reversible single-crystal-tosingle-crystal (SCSC) phase transitions. After the microscopic observations and crystallographic studies, it was revealed that the global similarity of the packing patterns of both phases with local differences, such as molecular stacking sequence and orientations, is the origin of the self-healing behavior of these crystals.

\section{Conclusions and perspectives}

Organic cocrystals have gained increasing interest as an emerging field of research, which alloys two different molecules together, providing a higher degree of freedom for achieving 
many novel physical properties. In this review, we focus on the application of organic cocrystals in the field of stimuliresponse. The natural characteristics of organic cocrystals include different molecular compositions, clear molecular packing modes, multiple intermolecular interactions, and electronic delocalization between $\mathrm{D}-\mathrm{A}$ molecules, which makes this material an ideal system for designing and application in rich stimuli-responsive functions. In the first section, the molecular packing mode of cocrystals, the main preparation methods, and the common donor and acceptor molecules in the cocrystal are briefly introduced. These basic knowledge of organic cocrystals is fundamental to understanding and designing its functional applications. Subsequently, the functional properties of organic cocrystals under external stimuli are highlighted, with emphasis on mechanical forces, solvents, acid-base, light and heat stimuli.

In general, the application of organic cocrystals in the field of stimuli response is still in its early stages and the amount of related research work reported is still limited. Therefore, it was highly necessary to provide an outlook on future developments in this field. (1) In cocrystal assembly, the monomer with stimuli-responsive behaviors could be selected. The benefit is that the stimuli-responsive property of the monomer will be retained after cocrystallization and a richer function is expected to be emerged. (2) The charge transfer between the donor and acceptor molecules in the organic cocrystals, and the resulting spatial electron delocalization, directly determines the extrinsic properties such as photoelectric properties of the material, considering that the regulation of external stimuli to adjust the degree of CT in cocrystals could stand as an effective means towards the design and incorporation of stimuli-responsive functions. (3) The relationship between internal molecular stacking structure and response property needs to be further understood, which will guide the rational design and synthesis of organic charge transfer cocrystals for stimuli-responsive applications.

In conclusion, the unique advantages of organic cocrystals have gradually opened up new research pathways for usage in stimuli-responsive applications. With increasing research interest towards this field, it is believed that more significant and interesting works will be explored and reported, thereby fostering deep understanding and promoting the development of organic cocrystals in stimuli-responsive fields.

\section{Conflicts of interest}

There are no conflicts to declare.

\section{Acknowledgements}

The authors acknowledge financial support from the Ministry of Science and Technology of China (Grants 2017YFA0204503, 2016YFB0401100), the National Natural Science Foundation of China (51702297, 51633006, 91833306, 51725304, 51733004, 51703159).

\section{Notes and references}

1 M. A. C. Stuart, W. T. Huck, J. Genzer, M. Müller, C. Ober, M. Stamm, G. B. Sukhorukov, I. Szleifer, V. V. Tsukruk and M. J. N. m. Urban, Emerging Applications of StimuliResponsive Polymer Materials, Nat. Mater., 2010, 9, 101.

2 Z. Chi, X. Zhang, B. Xu, X. Zhou, C. Ma, Y. Zhang, S. Liu and J. J. C. S. R. Xu, Recent Advances in Organic Mechanofluorochromic Materials, Chem. Soc. Rev., 2012, 41, 3878-3896.

3 X. Yan, F. Wang, B. Zheng and F. J. C. S. R. Huang, StimuliResponsive Supramolecular Polymeric Materials, Chem. Soc. Rev., 2012, 41, 6042-6065.

4 X. Zhang, L. Chen, K. H. Lim, S. Gonuguntla, K. W. Lim, D. Pranantyo, W. P. Yong, W. J. T. Yam, Z. Low, W. J. Teo, H. P. Nien, Q. W. Loh and S. Soh, The Pathway to Intelligence: Using Stimuli-Responsive Materials as Building Blocks for Constructing Smart and Functional Systems, Adv. Mater., 2019, 31, 1804540.

5 P. Galer, R. C. Korošec, M. Vidmar and B. Šket, Crystal Structures and Emission Properties of the $\mathrm{BF}_{2}$ Complex 1-Phenyl-3-(3,5-dimethoxyphenyl)-propane-1,3-dione: Multiple Chromisms, Aggregation- or Crystallization-Induced Emission, and the Self-Assembly Effect, J. Am. Chem. Soc., 2014, 136, 7383-7394.

6 Y. Dong, B. Xu, J. Zhang, X. Tan, L. Wang, J. Chen, H. Lv, S. Wen, B. Li, L. Ye, B. Zou and W. Tian, Piezochromic Luminescence Based on the Molecular Aggregation of 9,10-Bis((E)-2-(pyrid-2-yl)vinyl)anthracene, Angew. Chem., Int. Ed., 2012, 51, 10782-10785.

7 M. Morimoto and M. Irie, A Diarylethene Cocrystal that Converts Light into Mechanical Work, J. Am. Chem. Soc., 2010, 132, 14172-14178.

8 D.-K. Bučar and L. R. MacGillivray, Preparation and Reactivity of Nanocrystalline Cocrystals Formed via Sonocrystallization, J. Am. Chem. Soc., 2007, 129, 32-33.

9 P. Gupta, D. P. Karothu, E. Ahmed, P. Naumov and N. K. Nath, Thermally Twistable, Photobendable, Elastically Deformable, and Self-Healable Soft Crystals, Angew. Chem., Int. Ed., 2018, 130, 8634-8638.

10 Z. Fei, N. Kocher, C. J. Mohrschladt, H. Ihmels and D. Stalke, Single Crystals of the Disubstituted Anthracene 9,10- $\left(\mathrm{Ph}_{2} \mathrm{P}=\mathrm{S}\right)_{2} \mathrm{C}_{14} \mathrm{H}_{8}$ Selectively and Reversibly Detect Toluene by Solid-State Fluorescence Emission, Angew. Chem., Int. Ed., 2003, 42, 783-787.

11 Y. Zhen, K. Inoue, Z. Wang, T. Kusamoto and K. Nakabayashi, S.-i. Ohkoshi, W. Hu, Y. Guo, K. Harano and E. Nakamura, Acid-Responsive Conductive Nanofiber of Tetrabenzoporphyrin Made by Solution Processing, J. Am. Chem. Soc., 2018, 140, 62-65.

12 X.-L. Ni, S. Chen, Y. Yang and Z. Tao, Facile Cucurbit[8]uril-Based Supramolecular Approach To Fabricate Tunable Luminescent Materials in Aqueous Solution, J. Am. Chem. Soc., 2016, 138, 6177-6183.

13 G. Liu, J. Liu, X. Ye, L. Nie, P. Gu, X. Tao and Q. Zhang, SelfHealing Behavior in a Thermo-Mechanically Responsive 
Cocrystal during a Reversible Phase Transition, Angew. Chem., Int. Ed., 2017, 56, 198-202.

14 K. Isayama, N. Aizawa, J. Y. Kim and T. Yasuda, Modulating Photo- and Electroluminescence in a Stimuli-Responsive $\pi$-Conjugated Donor-Acceptor Molecular System, Angew. Chem., Int. Ed., 2018, 130, 12158-12162.

15 Y. Huang, Z. Wang, Z. Chen and Q. Zhang, Organic Cocrystals: Beyond Electrical Conductivities and FieldEffect Transistors (FETs), Angew. Chem., Int. Ed., 2019, 58, 9696-9711.

16 E. I. Kamitsos and W. M. Risen, Optically Induced Transformations of Metal TCNQ Materials, Solid State Commun., 1983, 45, 165-169.

17 C. Shi, Z. Guo, Y. Yan, S. Zhu, Y. Xie, Y. S. Zhao, W. Zhu and H. Tian, Self-assembly Solid-state Enhanced Red Emission of Quinolinemalononitrile: Optical Waveguides and Stimuli Response, ACS Appl. Mater. Interfaces, 2012, 5, 192-198.

18 J. Yang, Z. Ren, Z. Xie, Y. Liu, C. Wang, Y. Xie, Q. Peng, B. Xu, W. Tian, F. Zhang, Z. Chi, Q. Li and Z. Li, AIEgen with Fluorescence-Phosphorescence Dual Mechanoluminescence at Room Temperature, Angew. Chem., Int. Ed., 2017, 56, 880-884.

19 J. Yang, X. Gao, Z. Xie, Y. Gong, M. Fang, Q. Peng, Z. Chi and $\mathrm{Z}$. Li, Elucidating the Excited State of Mechanoluminescence in Organic Luminogens with Room-Temperature Phosphorescence, Angew. Chem., Int. Ed., 2017, 129, 15501-15505.

20 W. Z. Yuan, Y. Tan, Y. Gong, P. Lu, J. W. Y. Lam, X. Y. Shen, C. Feng, H. H. Y. Sung, Y. Lu, I. D. Williams, J. Z. Sun, Y. Zhang and B. Z. Tang, Synergy between Twisted Conformation and Effective Intermolecular Interactions: Strategy for Efficient Mechanochromic Luminogens with High Contrast, Adv. Mater., 2013, 25, 2837-2843.

21 P. Zhang, W. Dou, Z. Ju, X. Tang, W. Liu, C. Chen, B. Wang and W. Liu, Modularity Analysis of Tunable Solid-State Emission Based on a Twisted Conjugated Molecule Containing 9,9'-Bianthracene Group, Adv. Mater., 2013, 25, 6112-6116.

22 Y. Liu, Q. Zeng, B. Zou, Y. Liu, B. Xu and W. Tian, Piezochromic Luminescence of Donor-Acceptor Cocrystals: Distinct Responses to Anisotropic Grinding and Isotropic Compression, Angew. Chem., Int. Ed., 2018, 57, 15670-15674.

23 J. D. Wuest and Co-crystals Give Light, a Tune-Up, Nat, Chem, 2012, 4, 74-75.

24 W. Zhu, Y. Zhen, H. Dong, H. Fu and W. Hu, Organic Cocrystal Optoelectronic Materials and Devices, Prog. Chem., 2014, 26, 1292-1306.

25 X. Zhang, H. Dong and W. Hu, Organic Semiconductor Single Crystals for Electronics and Photonics, Adv. Mater., 2018, 30, 1801048.

26 L. Sun, Y. Wang, F. Yang, X. Zhang and W. Hu, Cocrystal Engineering: A Collaborative Strategy toward Functional Materials, Adv. Mater., 2019, 31, 1902328.

27 W. Zhu, L. Zhu, Y. Zou, Y. Wu, Y. Zhen, H. Dong, H. Fu, Z. Wei, Q. Shi and W. Hu, Deepening Insights of Charge
Transfer and Photophysics in a Novel Donor-Acceptor Cocrystal for Waveguide Couplers and Photonic Logic Computation, Adv. Mater., 2016, 28, 5954-5962.

28 K. P. Goetz, D. Vermeulen, M. E. Payne, C. Kloc, L. E. McNeil and O. D. Jurchescu, Charge-Transfer Complexes: New Perspectives on an Old Class of Compounds, J. Mater. Chem. C, 2014, 2, 3065-3076.

29 A. Khan, M. Wang, R. Usman, H. Sun, M. Du and C. Xu, Molecular Marriage via Charge Transfer Interaction in Organic Charge Transfer Co-Crystals toward Solid-State Fluorescence Modulation, Cryst. Growth Des., 2017, 17, 1251-1257.

30 L. Sun, W. Zhu, F. Yang, B. Li, X. Ren, X. Zhang and W. Hu, Molecular Cocrystals: Design, Charge-Transfer and Optoelectronic Functionality, Phys. Chem. Chem. Phys., 2018, 20, 6009-6023.

31 R. Bhowal, S. Biswas, A. Thumbarathil, A. L. Koner and D. Chopra, Exploring the Relationship between Intermolecular Interactions and Solid-State Photophysical Properties of Organic Co-Crystals, J. Phys. Chem. C, 2019, 123, 9311-9322.

32 J. Ferraris, D. O. Cowan, V. Walatka and J. H. Perlstein, Electron Transfer in a New Highly Conducting DonorAcceptor Complex, J. Am. Chem. Soc., 1973, 95, 948-949.

33 G. Bolla, H. Dong, Y. Zhen, Z. Wang and W. Hu, Organic Cocrystals: the Development of Ferroelectric Properties, Sci. China: Chem., 2016, 59, 523-530.

34 A. S. Tayi, A. K. Shveyd, A. C. H. Sue, J. M. Szarko, B. S. Rolczynski, D. Cao, T. J. Kennedy, A. A. Sarjeant, C. L. Stern, W. F. Paxton, W. Wu, S. K. Dey, A. C. Fahrenbach, J. R. Guest, H. Mohseni, L. X. Chen, K. L. Wang, J. F. Stoddart and S. I. Stupp, Room-Temperature Ferroelectricity in Supramolecular Networks of ChargeTransfer Complexes, Nature, 2012, 488, 485-489.

35 L. Sun, W. Zhu, W. Wang, F. Yang, C. Zhang, S. Wang, X. Zhang, R. Li, H. Dong and W. Hu, Intermolecular Charge-Transfer Interactions Facilitate Two-Photon Absorption in Styrylpyridine-Tetracyanobenzene Cocrystals, Angew. Chem., Int. Ed., 2017, 56, 7831-7835.

36 W. Zhu, L. Zhu, L. Sun, Y. Zhen, H. Dong, Z. Wei and $\mathrm{W}$. $\mathrm{Hu}$, Uncovering the Intramolecular Emission and Tuning the Nonlinear Optical Properties of Organic Materials by Cocrystallization, Angew. Chem., Int. Ed., 2016, 55, 14023-14027.

37 J. Zhang, H. Geng, T. S. Virk, Y. Zhao and J. Tan, C.-a. Di, W. Xu, K. Singh, W. Hu, Z. Shuai, Y. Liu and D. Zhu, SulfurBridged Annulene-TCNQ Co-Crystal: A Self-Assembled Molecular Level Heterojunction with Air Stable Ambipolar Charge Transport Behavior, Adv. Mater., 2012, 24, 2603-2607.

38 S. K. Park, S. Varghese, J. H. Kim, S.-J. Yoon, O. K. Kwon, B.-K. An, J. Gierschner and S. Y. Park, Tailor-Made Highly Luminescent and Ambipolar Transporting Organic Mixed Stacked Charge-Transfer Crystals: An Isometric Donor-Acceptor Approach, J. Am. Chem. Soc., 2013, 135, 4757-4764.

39 L. Sun, W. Hua, Y. Liu, G. Tian, M. Chen, M. Chen, F. Yang, S. Wang, X. Zhang, Y. Luo and W. Hu, Thermally Activated 
Delayed Fluorescence in an Organic Cocrystal: Narrowing the Singlet-Triplet Energy Gap via Charge Transfer, Angew. Chem., Int. Ed., 2019, 58, 11311-11316.

40 O. Bolton, K. Lee, H.-J. Kim, K. Y. Lin and J. J. N. c. Kim, Activating Efficient Phosphorescence from Purely Organic Materials by Crystal Design, Nat. Chem., 2011, 3, 205.

41 D. Vermeulen, L. Y. Zhu, K. P. Goetz, P. Hu, H. Jiang, C. S. Day, O. D. Jurchescu, V. Coropceanu, C. Kloc and L. E. McNeil, Charge Transport Properties of PeryleneTCNQ Crystals: The Effect of Stoichiometry, J. Phys. Chem. C, 2014, 118, 24688-24696.

42 J. Zhang, W. Xu, P. Sheng, G. Zhao and D. Zhu, Organic Donor-Acceptor Complexes as Novel Organic Semiconductors, Acc. Chem. Res., 2017, 50, 1654-1662.

43 H. Zhang, L. Jiang, Y. Zhen, J. Zhang, G. Han, X. Zhang, X. Fu, Y. Yi, W. Xu, H. Dong, W. Chen, W. Hu and D. Zhu, Organic Cocrystal Photovoltaic Behavior: A Model System to Study Charge Recombination of C-60 and C-70 at the Molecular Level, Adv. Electron. Mater., 2016, 2, 1500423.

44 Y. Sun, Y. Lei, L. Liao and W. Hu, Competition between Arene-Perfluoroarene and Charge-Transfer Interactions in Organic Light-Harvesting Systems, Angew. Chem., Int. Ed., 2017, 56, 10352-10356.

45 L. B. Coleman, M. J. Cohen, D. J. Sandman, F. G. Yamagishi, A. F. Garito and A. J. Heeger, Superconducting fluctuations and the peierls instability in an organic solid, Solid State Commun., 1973, 12, 1125-1132.

46 H. Jiang, P. Hu, J. Ye, K. K. Zhang, Y. Long, W. Hu and C. Kloc, Tuning of the Degree of Charge Transfer and the Electronic Properties in Organic Binary Compounds by Crystal Engineering: a Perspective, J. Mater. Chem. C, 2018, 6, 1884-1902.

47 F. X. Yang, S. S. Cheng, X. T. Zhang, X. C. Ren, R. J. Li, H. L. Dong and W. P. Hu, 2D Organic Materials for Optoelectronic Applications, Adv. Mater., 2018, 30, 1702415.

48 R. Li, W. Hu, Y. Liu and D. Zhu, Micro-and Nanocrystals of Organic Semiconductors, Acc. Chem. Res., 2010, 43, 529-540.

$49 \mathrm{H}$. Jiang and W. Hu, The Emergence of Organic SingleCrystal Electronics, Angew. Chem., Int. Ed., 2019, 56, 1408.

50 H. Jiang, P. Hu, J. Ye, Y. Li, H. Li, X. Zhang, R. Li, H. Dong, W. Hu and C. Kloc, Molecular Crystal Engineering: Tuning Organic Semiconductor from p-type to n-type by Adjusting Their Substitutional Symmetry, Adv. Mater., 2017, 29, 1605053.

51 H. Jiang, P. Hu, J. Ye, R. Ganguly, Y. Li, Y. Long, D. Fichou, W. Hu and C. Kloc, Hole Mobility Modulation in SingleCrystal Metal Phthalocyanines by Changing the Metal $-\pi / \pi-\pi$ Interactions, Angew. Chem., 2018, 130, 10269-10274.

52 H. Jiang, P. Hu, J. Ye, A. Chaturvedi, K. K. Zhang, Y. Li, Y. Long, D. Fichou, C. Kloc and W. Hu, From Linear to Angular Isomers: Achieving Tunable Charge Transport in Single-Crystal Indolocarbazoles Through Delicate Synergetic $\mathrm{CH} / \mathrm{NH} \cdots \pi$ Interactions, Angew. Chem., Int. Ed., 2018, 57, 8875-8880.

53 J. Zhang, J. Jin, H. Xu, Q. Zhang and W. Huang, Recent Progress on Organic Donor-Acceptor Complexes as Active
Elements in Organic Field-Effect Transistors, J. Mater. Chem. C, 2018, 6, 3485-3498.

54 B. Wang, S. Zheng, A. Saha, L. Bao, X. Lu and D. M. Guldi, Understanding Charge-Transfer Characteristics in Crystalline Nanosheets of Fullerene/(Metallo)porphyrin Cocrystals, J. Am. Chem. Soc., 2017, 139, 10578-10584.

55 W. Zhu, R. Zheng, Y. Zhen, Z. Yu, H. Dong, H. Fu, Q. Shi and $\mathrm{W}$. $\mathrm{Hu}$, Rational Design of Charge-Transfer Interactions in Halogen-Bonded Co-Crystals toward Versatile Solid-State Optoelectronics, J. Am. Chem. Soc., 2015, 137, 11038-11046.

56 J. Zhang, J. Tan, Z. Ma, W. Xu, G. Zhao and H. Geng, C. a. Di, W. Hu, Z. Shuai, K. Singh and D. Zhu, Fullerene/SulfurBridged Annulene Cocrystals: Two-Dimensional Segregated Heterojunctions with Ambipolar Transport Properties and Photoresponsivity, J. Am. Chem. Soc., 2013, 135, 558-561.

57 N. Blagden, D. J. Berry, A. Parkin, H. Javed, A. Ibrahim, P. T. Gavan, L. L. De Matos and C. C. Seaton, Current Directions in Co-Crystal Growth, New J. Chem., 2008, 32, 1659-1672.

58 P. Zhang, G. Bolla, G. Qiu, Z. Shu, Q. Yan, Q. Li, S. Ding, Z. Ni, W. Zhu, H. Dong, Y. Zhen and W. Hu, Halogen bonded cocrystal polymorphs of 1,4-di(4-pyridyl)-1,3diacetylene, CrystEngComm, 2017, 19, 4505-4509.

59 Y. Qin, C. Cheng, H. Geng, C. Wang, W. Hu, W. Xu, Z. Shuai and D. Zhu, Efficient Ambipolar Transport Properties in Alternate Stacking Donor-Acceptor Complexes: from Experiment to Theory, Phys. Chem. Chem. Phys., 2016, 18, 14094-14103.

$60 \mathrm{P} . \mathrm{Hu}, \mathrm{K} . \mathrm{Du}, \mathrm{F}$. Wei, H. Jiang and C. Kloc, Crystal Growth, HOMO-LUMO Engineering, and Charge Transfer Degree in Perylene-FxTCNQ ( $x=1,2$, and 4) Organic Charge Transfer Binary Compounds, Cryst. Growth Des., 2016, 16, 3019-3027.

61 P. Hu, H. Li, Y. Li, H. Jiang and C. Kloc, Single-crystal growth, structures, charge transfer and transport properties of anthracene- $\mathrm{F}_{4}$ TCNQ and tetracene- $\mathrm{F}_{4}$ TCNQ chargetransfer compounds, CrystEngComm, 2017, 19, 618-624.

62 X. Ye, Y. Liu, Q. Guo, Q. Han, C. Ge, S. Cui, L. Zhang and $\mathrm{X}$. Tao, 1D versus 2D cocrystals Growth via Microspacing In-Air Sublimation, Nat. Commun., 2019, 10, 761.

63 W. Zhu, Y. Yi, Y. Zhen and W. Hu, Precisely Tailoring the Stoichiometric Stacking of Perylene-TCNQ Co-Crystals towards Different Nano and Microstructures with Varied Optoelectronic Performances, Small, 2015, 11, 2150-2156.

64 D. Braga, L. Maini and F. Grepioni, Mechanochemical Preparation of Co-Crystals, Chem. Soc. Rev., 2013, 42, 7638-7648.

65 T. Friščić and W. Jones, Recent Advances in Understanding the Mechanism of Cocrystal Formation via Grinding, Cryst. Growth Des., 2009, 9, 1621-1637.

66 T. Hasegawa, K. Mattenberger, J. Takeya and B. Batlogg, Ambipolar Field-Effect Carrier Injections in Organic Mott Insulators, Phys. Rev. B: Condens. Matter Mater. Phys., 2004, 69, 245115. 
67 M. Sakai, H. Sakuma, Y. Ito, A. Saito, M. Nakamura and K. Kudo, Ambipolar Field-Effect Transistor Characteristics of (BEDT-TTF)(TCNQ) Crystals and Metal-like Conduction Induced by a Gate Electric Field, Phys. Rev. B: Condens. Matter Mater. Phys., 2007, 76, 045111.

68 K. P. Goetz, J. Tsutsumi, S. Pookpanratana, J. Chen, N. S. Corbin, R. K. Behera, V. Coropceanu, C. A. Richter, C. A. Hacker, T. Hasegawa and O. D. Jurchescu, Polymorphism in the 1:1 Charge-Transfer Complex DBTTF-TCNQ and Its Effects on Optical and Electronic Properties, Adv. Electron. Mater., 2016, 2, 1600203.

69 Y. Wang, W. Zhu, W. Du, X. Liu, X. Zhang, H. Dong and W. Hu, Cocrystals Strategy towards Materials for NearInfrared Photothermal Conversion and Imaging, Angew. Chem., Int. Ed., 2018, 57, 3963-3967.

70 Y. L. Lei, L. S. Liao and S. T. Lee, Selective Growth of DualColor-Emitting Heterogeneous Microdumbbells Composed of Organic Charge-Transfer Complexes, J. Am. Chem. Soc., 2013, 135, 3744-3747.

71 Y.-Q. Sun, Y.-L. Lei, X.-H. Sun, S.-T. Lee and L.-S. Liao, Charge-Transfer Emission of Mixed Organic Cocrystal Microtubes over the Whole Composition Range, Chem. Mater., 2015, 27, 1157-1163.

72 Y. Wang, Y. Li, W. Zhu, J. Liu, X. Zhang, R. Li, Y. Zhen, H. Dong and W. Hu, Co-Crystal Engineering: a Novel Method to Obtain One-Dimensional (1D) Carbon Nanocrystals of Corannulene-Fullerene by a Solution Process, Nanoscale, 2016, 8, 14920-14924.

73 H.-D. Wu, F.-X. Wang, M. Zhang and G.-B. Pan, Investigation of Transport Properties of Coronene-TCNQ Cocrystal Microrods with Coronene Microrods and TCNQ Microsheets, Nanoscale, 2015, 7, 12839-12842.

74 W. Zhu, R. Zheng, X. Fu, H. Fu, Q. Shi, Y. Zhen, H. Dong and W. Hu, Revealing the Charge-Transfer Interactions in SelfAssembled Organic Cocrystals: Two-Dimensional Photonic Applications, Angew. Chem., Int. Ed., 2015, 54, 6785-6789.

75 M.-P. Zhuo, Y.-C. Tao, X.-D. Wang, Y. Wu, S. Chen, L.-S. Liao and L. Jiang, 2D Organic Photonics: An Asymmetric Optical Waveguide in Self-Assembled Halogen-Bonded Cocrystals, J. Am. Chem. Soc., 2018, 130, 11470-11474.

76 S. d'Agostino, F. Grepioni, D. Braga and B. Ventura, Tipping the Balance with the Aid of Stoichiometry: Room Temperature Phosphorescence versus Fluorescence in Organic Cocrystals, Cryst. Growth Des., 2015, 15, 2039-2045.

77 S. K. Park, J. H. Kim, T. Ohto, R. Yamada, A. O. F. Jones, D. R. Whang, I. Cho, S. Oh, S. H. Hong, J. E. Kwon, J. H. Kim, Y. Olivier, R. Fischer, R. Resel, J. Gierschner, H. Tada and S. Y. Park, Highly Luminescent 2D-Type Slab Crystals Based on a Molecular Charge-Transfer Complex as Promising Organic Light-Emitting Transistor Materials, Angew. Chem., Int. Ed., 2017, 29, 1701346.

78 C. Karunatilaka, D.-K. Bučar, L. R. Ditzler, T. Friščić, D. C. Swenson, L. R. MacGillivray and A. V. Tivanski, Softening and Hardening of Macro- and Nano-Sized Organic Cocrystals in a Single-Crystal Transformation, Angew. Chem., Int. Ed., 2011, 50, 8642-8646.
79 S. Li and D. Yan, Tuning Light-Driven Motion and Bending in Macroscale-Flexible Molecular Crystals Based on a Cocrystal Approach, ACS Appl. Mater. Interfaces, 2018, 10, 22703-22710.

80 M. Krikorian, S. Liu and T. M. Swager, Columnar liquid crystallinity and mechanochromism in cationic platinum (II) complexes, J. Am. Chem. Soc., 2014, 136, 2952-2955.

81 S. Xu, T. Liu, Y. Mu, Y. F. Wang, Z. Chi, C. C. Lo, S. Liu, Y. Zhang, A. Lien and J. Xu, An Organic Molecule with Asymmetric Structure Exhibiting Aggregation-Induced Emission, Delayed Fluorescence, and Mechanoluminescence, Angew. Chem., Int. Ed., 2015, 54, 874-878.

82 Z. Ma, M. Teng, Z. Wang, S. Yang and X. Jia, Mechanically Induced Multicolor Switching Based on a Single Organic Molecule, Angew. Chem., Int. Ed., 2013, 52, 12268-12272.

83 D. Yan, H. Yang, Q. Meng, H. Lin and M. Wei, TwoComponent Molecular Materials of 2,5-Diphenyloxazole Exhibiting Tunable Ultraviolet/Blue Polarized Emission, Pump-enhanced Luminescence, and Mechanochromic Response, Adv. Funct. Mater., 2014, 24, 587-594.

84 X. Luo, J. Li, C. Li, L. Heng, Y. Q. Dong, Z. Liu, Z. Bo and B. Z. Tang, Reversible Switching of the Emission of Diphenyldibenzofulvenes by Thermal and Mechanical Stimuli, Adv. Mater., 2011, 23, 3261-3265.

85 Y. Sagara, S. Yamane, M. Mitani, C. Weder and T. Kato, Mechanoresponsive Luminescent Molecular Assemblies: An Emerging Class of Materials, Adv. Mater., 2016, 28, 1073-1095.

86 Y. Wang, X. Tan, Y.-M. Zhang, S. Zhu, I. Zhang, B. Yu, K. Wang, B. Yang, M. Li, B. Zou and S. X.-A. Zhang, Dynamic Behavior of Molecular Switches in Crystal under Pressure and Its Reflection on Tactile Sensing, J. Am. Chem. Soc., 2015, 137, 931-939.

87 J. Luo, L. Y. Li, Y. Song and J. Pei, A Piezochromic Luminescent Complex: Mechanical Force Induced Patterning with a High Contrast Ratio, Chem. - Eur. J., 2011, 17, 10515.

88 V. W.-W. Yam, V. K.-M. Au and S. Y.-L. Leung, LightEmitting Self-Assembled Materials Based on d8 and d10 Transition Metal Complexes, Chem. Rev., 2015, 115, 7589-7728.

89 Y. Zhang, Q. Song, K. Wang, W. Mao, F. Cao, J. Sun, L. Zhan, Y. Lv, Y. Ma, B. Zou and C. Zhang, Polymorphic Crystals and Their Luminescence Switching of Triphenylacrylonitrile Derivatives upon Solvent Vapour, Mechanical, and Thermal stimuli, J. Mater. Chem. C, 2015, 3, 3049-3054.

90 L. Wang, K. Wang, B. Zou, K. Ye, H. Zhang and Y. Wang, Luminescent Chromism of Boron Diketonate Crystals: Distinct Responses to Different Stresses, Adv. Mater., 2015, 27, 2918-2922.

91 S. Dey, S. Das, S. Bhunia, R. Chowdhury, A. Mondal, B. Bhattacharya, R. Devarapalli, N. Yasuda, T. Moriwaki, K. Mandal, G. D. Mukherjee and C. M. Reddy, Mechanically Interlocked Architecture Aids an Ultra-Stiff and UltraHard Elastically Bendable Cocrystal, Nat. Commun., 2019, 10, 3711. 
92 S. Ghosh and C. M. Reddy, Elastic and Bendable Caffeine Cocrystals: Implications for the Design of Flexible Organic Materials, Angew. Chem., Int. Ed., 2012, 51, 10319-10323.

93 G. Liu, J. Liu, Y. Liu and X. Tao, Oriented Single-Crystal-toSingle-Crystal Phase Transition with Dramatic Changes in the Dimensions of Crystals, J. Am. Chem. Soc., 2014, 136, 590-593.

94 J. Liu, G. Liu, Y. Liu, X. Zheng, Q. Han, X. Ye and X. Tao, The Role of Weak Interactions in the Mechano-induced Single-Crystal-to-Single-Crystal Phase Transition of 8-Hydroxyquinoline-Based Co-Crystals, Chem. - Asian J., 2016, 11, 1682-1687.

95 P. Naumov, S. Chizhik, M. K. Panda, N. K. Nath and E. Boldyreva, Mechanically Responsive Molecular Crystals, Chem. Rev., 2015, 115, 12440-12490.

96 B. Lu, Y. Zhang, X. Yang, K. Wang, B. Zou and D. Yan, Piezochromic Luminescence of AIE-Active Molecular Co-Crystals: Tunable Multiple Hydrogen Bonding and Molecular Packing, J. Mater. Chem. C, 2018, 6, 9660-9666.

97 J. Wang, A. Li, S. Xu, B. Li, C. Song, Y. Geng, N. Chu, J. He and $\mathrm{W}$. $\mathrm{Xu}$, Tunable Luminescence of a Novel Organic Co-Crystal based on Intermolecular Charge Transfer under Pressure, J. Mater. Chem. C, 2018, 6, 8958-8965.

98 S. K. Park, I. Cho, J. Gierschner, J. H. Kim, J. H. Kim, J. E. Kwon, O. K. Kwon, D. R. Whang, J.-H. Park, B.-K. An and S. Y. Park, Stimuli-Responsive Reversible Fluorescence Switching in a Crystalline Donor-Acceptor Mixture Film: Mixed Stack Charge-Transfer Emission versus Segregated Stack Monomer Emission, Angew. Chem., Int. Ed., 2016, 55, 203-207.

99 H. Sun, M. Wang, X. Wei, R. Zhang, S. Wang, A. Khan, R. Usman, Q. Feng, M. Du, F. Yu, W. Zhang and C. Xu, Understanding Charge-Transfer Interaction Mode in Cocrystals and
Solvates of 1-Phenyl-3-(pyren-1-yl) Prop-2-en-1-one and TCNQ, Cryst. Growth Des., 2015, 15, 4032-4038.

100 S. P. Anthony, S. Varughese and S. M. Draper, Switching and tuning organic solid-state luminescence via a supramolecular approach, Chem. Commun., 2009, 7500-7502.

101 J. Zhang, G. Liu, Y. Zhou, G. Long, P. Gu and Q. Zhang, Solvent Accommodation: Functionalities Can Be Tailored Through Co-Crystallization Based on 1:1 Coronene-F4TCNQ Charge-Transfer Complex, ACS Appl. Mater. Interfaces, 2017, 9, 1183-1188.

102 Y. Sun, Y. Lei, H. Dong, Y. Zhen and W. Hu, Solvatomechanical Bending of Organic Charge Transfer Cocrystal, J. Am. Chem. Soc., 2018, 140, 6186-6189.

103 S. Li and D. Yan, A Reversible Acid-Base Fluorescent Switch based on Molecular Cocrystal of 4-[2-4-quinolinyl)vinyl] Phenol and Tetrafluoroterephthalic Acid, Sci. China: Chem., 2018, 61, 215-221.

104 S. Li and D. Yan, Two-Component Aggregation-Induced Emission Materials: Tunable One/Two-Photon Luminescence and Stimuli-Responsive Switches by Co-Crystal Formation, Adv. Opt. Mater., 2018, 6, 1800445.

105 H. Dong, H. Zhu, Q. Meng, X. Gong and W. Hu, Organic photoresponse materials and devices, Chem. Soc. Rev., 2012, 41, 1754-1808.

106 K. J. Baeg, M. Binda, D. Natali, M. Caironi and Y. Y. Noh, Organic Light Detectors: Photodiodes and Phototransistors, Adv. Mater., 2013, 25, 4267-4295.

107 Y. Wang, L. Sun, C. Wang, F. Yang, X. Ren, X. Zhang, H. Dong and W. Hu, Organic Crystalline Materials in Fexible Electronics, Chem. Soc. Rev., 2019, 48, 1492-1530.

108 O. S. Bushuyev, T. C. Corkery, C. J. Barrett and T. Friščić, Photo-Mechanical Azobenzene Cocrystals and In Situ X-ray diffraction Monitoring of Their Optically-Induced Crystalto-Crystal Isomerisation, Chem. Sci., 2014, 5, 3158-3164. 